\title{
Aus Fehlern lernen - Zeit für CIRS
}

Fine offene Fehlerkultur wird im deutEschen Gesundheitswesen noch nicht überall gelebt. Um künftig Behandlungsfehler zu minimieren sowie die Sicherheit für Patienten und den Qualitätsstandard zu erhöhen, will der Berufsverband der Deutschen Urologen (BDU) e.V. die Einführung eines Fehlermanagementsystems in der Urologie vorantreiben. Auf Basis des Critical Incident Reporting Systems (CIRS), in dessen Netzwerk CIRSmedical. de bereits über 30 Berichtsgruppen (z.B. Chirurgen, Kinderärzte, Hämatoonkologen) vertreten sind, soll die fachspezifische Variante CIRS-Urologie als anonymes, internetbasiertes Berichts- und Lernsystem für kritische Ereignisse und Fehler in der Medizin auch im ambulanten Bereich implementiert werden. CIRS wird vom Ärztlichen Zentrum für Qualität in der Medizin (ÄZQ) betreut. Politischen Forderungen nach verpflichtenden Registern bei medi- zinischen Fehlern und Zwischenfällen tritt der BDU mit der Einführung von CIRSUrologie damit entgegen. Seit dem 1. November kann das System mit Fehlern gespeist werden.

Dr. Wolfgang Bühmann, Pressesprecher des BDU, der das Konzept bei der Eröffnungspressekonferenz am 27.9. vorstellte, erklärte, dass die freiwillige Teilnahme, die Anonymität und die Sanktionsfreiheit Voraussetzungen dafür sind, damit eine Fehler- und Sicherheitskultur überhaupt entstehen kann. Fehler dürfen nach seinen Worten nicht länger als Makel interpretiert werden, sondern als Chance, besser zu werden, sicherheitsrelevante Ereignisse zu erkennen und Strategien zur Fehlervermeidung zu entwickeln.

Mit CIRS werden Fehler, sei es in der Beschriftung einer Urinprobe oder in der Behandlung des Patienten selbst, kritische Ereignisse oder (Beinahe-)Schäden ver- schlüsselt über ein spezifisches OnlineFormular an das CIRS-Team des ÄZQ gesendet. Fachleute des CIRS-Teams Urologie analysieren und kommentieren diese Berichte. Ihr Feedback steht dann den Berichtenden und allen anderen Nutzern im Internet als „Kasuistik zum Lernen“ zur Verfügung.

Dr. Claudia Mäck

Eröffnungspressekonferenz

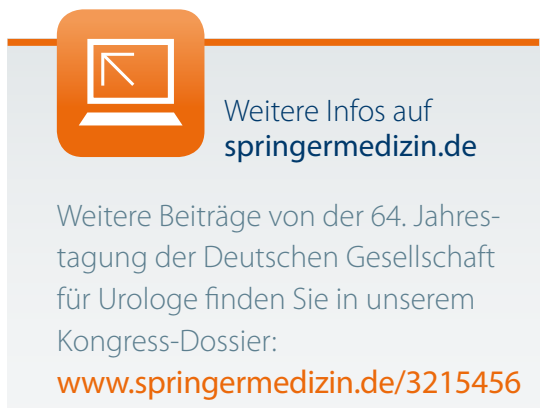

